\title{
Spatial inference using networks of RFID receiver: a Bayesian approach
}

\author{
Ying Zhu ${ }^{1}$ William Howard ${ }^{2}$ Ken Q. Pu ${ }^{2}$ \\ ${ }^{1}$ Faculty of Business and IT, ${ }^{2}$ Faculty of Science \\ University of Ontario Inst. of Technology \\ 2000 Simcoe Street N. \\ Oshawa, ON, Canada, L1H 7K4
}

\begin{abstract}
We introduce a statistical technique of inferring the position of RFID tags by means of computation on multiple data streams from distributed RFID receivers. Using a single statistical model that describes the detection rate between tags and receivers, we are able to perform Bayesian inference on the positions of the observed tags. If permitted by the receivers, our method can optimally coordinate the signal strength of multiple receivers to improve the time response of our positional inference system.
\end{abstract}

\section{INTRODUCTION}

Radio frequency identification (RFID) technology has rapidly been adopted as an innovation in inventory management. Most noticable success stories of RFID deployment have been Walmart inventory tracking system [5], and airline baggage tracking systems [3]. RFID systems is seen as a standard component in supply chain management [7], [11], and is seen as a potential technology for many areas of applications [2], [9].

The key feature offered by the RFID technolgy is its ability to automate tagging individual items and sense their presence at key locations. For example, Gillete razors have been equipped with RFID tags [4], and thus tracks the sales in realtime in all point of sales that have receivers installed. The ability track individual items lies in the fact that RFID tags are extremely pervasive and can carry enough bits to uniquely identify individual items. Furthermore, RFID readers can simultaneously detect large number of RFID tags present.

Despite the success, RFID technology presents challenges when used for more complex monitoring beyond the simple presence-absence detection. Many researchers have identified and offered solutions to issues such as false reading [11] and interference [2]. In this paper, we address the challenge of inferring spatial information of detected RFID tags by aggregation of data streams from a network of RFID receivers. In contrast to the existing localization methods [6], [1], [10], [8], our spatial inference technique offers the following features:

- We do not assume any special capabilities of the RFID readers. Thus, our method applies to both active and passive readers. It is not even necessary for the readers to offer signal strength information.

- We do not assume any model of the signal interference. Our method is highly robust to even very noisy and heterogenous environments.

- We provide active tracking capability. Our method allows one to actively control the RFID readers to cooperatively track a particular tag in order to speed up the inference response time.

Our contributions are:

- A model based framework for spatial inference of RFID tags is introduced using Bayesian inference.

- The framework is generalized to work for RFID receivers that do not provide signal strength information, but can make use if the signal strengh information if it is available.

- The framework allows optimal active tracking by controlling multiple RFID receivers in a coordinated fashion.

\section{BACKGROUND AND MOTIVATION}

We are interested at the problem of inferring the spatial location of RFID tags by aggregating multiple data streams from a network of receivers. RFID technology comes in two families: active and passive. In active RFID system, tags are battery powered, and emit radio frequency signals. Active RFID receivers simply pick up the radio signals and decode the digital data carried by the respective tag. The advantages of active RFID systems are:

- The range of active RFID system is significantly larger than passive counter parts.

- Active RFID tags can carry dynamic data such as temperature readings.

However, the disadvantage of active RFID technology is that tags are bulky, requiring battery pack, and are magnitudes more expensive than passive tags.

In passive RFID systems, receivers transmits an outgoing radio signal which will excite the microscopic antenna of the passive tags. The passive RFID tags uses the radio signal to power an onboard chip and retransmit an echo signal that carries digital information. The advantage of passive RFID system is that tags are very pervasive and extremely inexpensive. However, the range of passive RFID systems is limited and the data carried by passive tags must be static.

Common to both active and passive RFID systems, the receivers can be configured to different power levels. For 
active receivers, one can change the sensitivity of its antenna, thus effectively changing its range of coverage. For passive receivers, one can set the power level of the outgoing antenna to achieve similar effect. Furthermore, many of the receivers make the signal strength of the detected tags available to the user. It is these two features of RFID receivers that allow one to compute the spatial information of RFID tags. It is important to note that some models of RFID receivers do not provide signal strength. Thus, one of our objective is to develop spatial inference techniques that work even when signal strength is not available.

A well-known practical issue with RFID deployment is interference and obstruction of radio signals. Hetergenous environmental obstructions can severely limit the detection of tags by the receivers. From the perspective of spatial localization, one must compensate for such spatial heterogeneity due to radio interference, reflection and obstruction. Our objective is to adapt to such signal irregularities without user defined models.

\section{RELATED WORK}

The value of spatial information of RFID tags can range from being useful to being extremely valuable to being absolutely necessary [5], [2]. However, common RFID tags and receivers do not provide any spatial information. Thus, the problem of localization and infering spatial information has been the subject of research.

$\mathrm{Ni}$ et al described the LANDMARC [8] location sensing system for active RFIDs. Their system uses signal strength and a set of rules to determine the location of active RFID tags. Chen and Lee described how the similar rule-based approach can also be applied to WiFi networks [1]. Lee and Chen [6] also proposed a localization method for WiFi networks. with the knowledge of a predefined spatial model. Since active RFID receivers act similarly to $\mathrm{WiFi}$ antennas, their modelbased approach should also be applicable to active RFID localization. Thiem et al [10] proposed a localization of RFID tags in heterogeneous mesh networks.

Our work extends the existing localization method in several ways. We do not explicitly assume any fixed model. By a model, we mean a statistical model that describes the signal characteristics as a function of spatial separation between the receiver and the tag. The statistical model is obtained automatically from the calibration phase, so no user knowledge is required to construct the model parameters. In our framework, localization is done by means of statistical inference, so not only do we infer the most likely position of the tag, but we actual obtain the likelihood of the tag at all known positions. This is great if the user wishes to compute the top- $k$ likely positions of a tag. Our work also naturally accomodates active and passive tags using different statistical models. Finally, within the formalism of Bayesian inference, we naturally permit user-defined prior knowledge of where the tags should be found.
$\mathbf{T}$ is the set of tags.

$t \in \mathbf{T}$ is a specific tag.

$\mathbf{X}_{\text {cali }}$ is the set of calibration positions.

$\mathbf{x} \in \mathbf{X}_{\text {cali }}$ is a specific location.

$D(t)$ is the event that tag $t$ is detected in the data stream.

$\mathbf{R}$ is the set of receivers in the network.

$R \in \mathbf{R}$ is a specific receiver in the network.

$\operatorname{PL}(R)$ is the power level of receiver $R$.

$s$ is the signal strength.

Fig. 1. Notations used throughout the paper

\section{MOdELED SPATIAL INFERENCE WITHOUT SIGNAL STRENGTH INFORMATION}

We treating the detection of tags by receivers as a stochastic process. The probability of detection is determined by the position of the tags, the detecting receiver and its power level.

Definition 1 (Statistical models): Given a tag $t$ and a receiver $R$. If the receiver does not provide signal strength data, then we assume that the detection of tag $t$ follows a Bernoulli process, with $\alpha(R, \mathbf{x}, L)=p(D(t) \mid R, \mathbf{x}, L)$ to be the probability of detecting tag $t$ located at position $\mathrm{x}$ by the receiver $R$ given that the receiver's power level is set to $L(R)$.

Denote the model as $\mathcal{M}$. Its model parameters are the set of $\alpha$ constants.

$$
\theta(\mathcal{M})=\left\{\alpha(R, \mathbf{x}, L): R \in \mathbf{R}, \mathbf{x} \in \mathbf{X}_{\text {cali }}, L \in \operatorname{PL}(R)\right\}
$$

As with all model based statistical methods, model parameter estimation is an important step towards inference. We obtain the model parameters $\theta(\mathcal{M})$ by simple calibration. With assign an arbitrarily chosen set of calibration positions, $\mathbf{X}_{\text {cali }}$, over the area of coverage. These points need not form a regular grid. We then place RFID tags at each position and monitor the data streams from all RFID receivers.

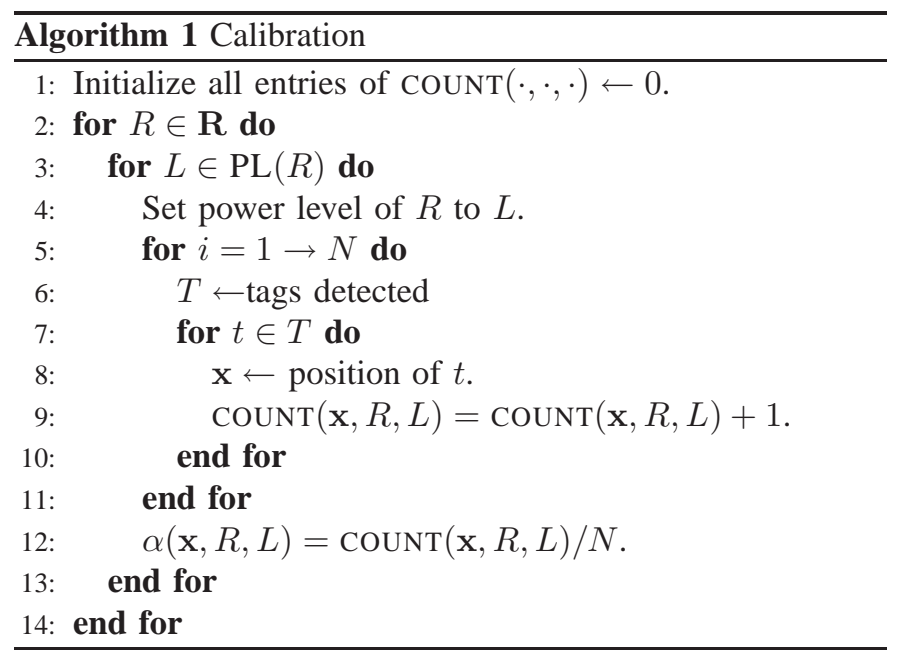

Algorithm 1 outlines the algorithm used to estimate the model parameters. The algorithm instructs all receivers to sample $N$ times for each of its available power level. The probability of a tag $t$ of being detected depends on the 
position $\mathbf{x}$ of the tag $t$. This probability is estimated simply by $\operatorname{COUNT}(\mathbf{x}, R, L) / N$. It is worth noting that the calibration can be carried out concurrently by executing line 3 - line 12 in Algorithm 1 in separate parallel threads. More discussions on the implementation and experimental results on calibration are presented in Section VII.

Given the model and its estimated parameters, we are able to perform statistical inference on the position of tags based on the observations from the network of RFID receivers. During the spatial inference phase, we instruct each receiver $R \in \mathbf{R}$ to continuously provides a stream of detected tags in the form of:

$$
A=\begin{array}{|l|l|l|}
\hline \text { receiver } & \text { power level } & \text { detected tags } \\
\hline \cdots & \cdots & \cdots \\
\hline
\end{array}
$$

We refer to the table $A$ as the raw observations.

The raw observations are collected distributedly from the RFID receivers. We refer to the observation phase as tracking. A simple random tracking algorithm is described in Algorithm 2.

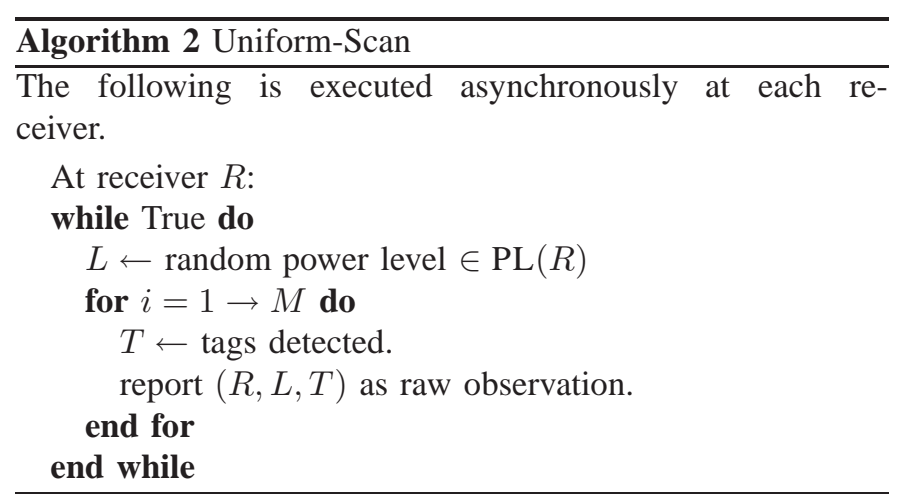

Given a specific tag $t$, in order to infer its most likely spatial position, we first aggregate the raw observations in $A$ by counting total number of positive detections of $t$ and the total number of detections made, grouped by the receivers and their power levels, thus producing the following table.

$B=$\begin{tabular}{|l|l|l|l|}
\hline receiver & power level & detections of $t$ & total detections made \\
\hline$R_{1}$ & $L_{1}$ & $D_{t}\left(R_{1}, L_{1}\right)$ & $N\left(R_{1}, L_{1}\right)$ \\
$\cdots$ & $\ldots$ & $\ldots$ & $\ldots$ \\
\hline
\end{tabular}

We denote $D_{t}(R, L)$ to be the number of times that tag $t$ has been detected by receiver $R$ at power level $L$, and $N(R, L)$ to be the total number of detections made by receiver $R$ at power level $L$. Collectively, we refer to data in table $B$ as the observation, denoted by OBSERV.

$$
\begin{aligned}
& p(\mathbf{x} \mid \text { ObSERV })=\frac{p(\text { OBSERV } \mid \mathbf{x}) p(\mathbf{x})}{p(\text { OBSERV })} \\
\propto & p(\text { OBSERV } \mid \mathbf{x}) p(\mathbf{x}), \text { since } p(\text { OBSERV }) \text { is a constant. } \\
= & \prod_{(R, L)} p\left(D_{t}(R, L) \mid \mathbf{x}, N(R, L)\right) p(N(R, L) \mid \mathbf{x}) p(\mathbf{x}) \\
= & \prod_{(R, L)} \mathbf{B}\left(D_{t}(R, L) \mid N(R, L), \alpha(\mathbf{x}, R, L)\right) p(N(R, L) \mid \mathbf{x}) p(\mathbf{x})
\end{aligned}
$$

where $\mathbf{B}$ is the Binomial distribution:

$$
\mathbf{B}(K \mid N, \alpha)=\left(\begin{array}{c}
N \\
K
\end{array}\right) \alpha^{K}(1-\alpha)^{N-K}
$$

The term $p(\mathbf{x})$ is the prior belief of where the tag $t$ should be. If the scenarios of warehouse tracking, the application may have some prior knowledge of where the tag must be, in which case, during the inference, the user application may optionally set $p(\mathbf{x})=0$ if it is for certain that tag $t$ cannot possibly be at position $\mathbf{x}$. If no prior knowledge is available, then $p(\mathbf{x})=$ constant $=1 /\left|\mathbf{X}_{\text {cali }}\right|$.

The term $p(N(R, L) \mid \mathbf{x})$ is the probability of the application receiving a $N(R, L)$ samples from receiver $R$ at power level $L$. This is determined by the tracking strategy. The random tracking algorithm (Algorithm 2) samples equal number of readings from all power levels from all receivers, thus $p(N(R, L) \mid \mathbf{x})=$ constant. Therefore, for random tracking, we get,

$$
\begin{aligned}
& p(\mathbf{x} \mid \text { OBSERV }) \\
= & c \prod_{R, L} \mathbf{B}\left(D_{t}(R, L) \mid N(R, L), \alpha(\mathbf{x}, R, L)\right) \cdot p(\mathbf{x})
\end{aligned}
$$

Equation 1 can be computed for each position $\mathbf{x}$ from the aggregated observation OBSERV and the prior distribution. The most likely position $\mathrm{x}^{*}$ is the one with the highest probability given the observation:

$$
\mathbf{x}^{*}=\arg \max _{\mathbf{x} \in \mathbf{X}_{\text {cali }}} p(\mathbf{x} \mid \text { OBSERV })
$$

\section{MOdel-BASED SPATIAL INFERENCE WITH SIGNAL STRENGTH}

Methods of calibration, tracking and inference can be generalized to take into consideration of signal strength. If the receiver provides the signal strength, then we assume that the distribution of the signal strength $s$ follows a Gaussian distribution. Thus,

$$
f(D(t)=s \mid \mathbf{x}, R, L)=\frac{1}{\sigma \sqrt{2 \pi}} \exp \left(-\frac{(s-\mu)^{2}}{2 \sigma^{2}}\right)
$$

where, $\mu=\mu(\mathbf{x}, R, L)$ and $\sigma=\sigma(\mathbf{x}, R, L)$.

The observations are of the form: $A=\left(R, L,\left\{\left(t_{i}, s_{i}\right)\right\}\right)$, where $\left(t_{i}, s_{i}\right)$ are the detected tag ID and the signal strength respectively.

For each $t$, let $\mathrm{x}$ be its location.

$$
\begin{gathered}
\mu(\mathbf{x}, R, L)=\frac{\sum_{R, L, t} \operatorname{SignAL}(t)}{|\{(R, L, t) \in A\}|} \\
\sigma(\mathbf{x}, R, L)^{2}=\frac{\sum_{R, L, t}\left(\operatorname{SignaL}(t)^{2}-\mu(\mathbf{x}, R, L)\right)}{|\{(R, L, t) \in A\}|}
\end{gathered}
$$

Note, $\mu$ and $\sigma^{2}$ are just the mean and variance of the observed signal strengths.

We note that all receivers have some imprecision $\pm \Delta s$, so a signal strength of $s$ actually represents a signal interval of $[s-\Delta s, s+\Delta s]$. Let $p(D(t)=s \mid \mathbf{x}, R, L)$ be the probability of observing signal strength $s$ when the location of the tag is $\mathbf{x}$ and the receiver $R$ is operating at power level $L$.

$$
p(D(t)=s \mid \mathbf{x}, R, L)=\int_{s-\Delta s}^{s+\Delta s} f(D(t)=s \mid \mathbf{x}, R, L) d s
$$


If we assume that the receiver is high precision, i.e. $\Delta s \ll \sigma$, then we can approximate the integral by

$$
p(D(t)=s \mid \mathbf{x}, R, L) \approx c \cdot f(D(t)=s \mid \mu, \sigma)
$$

for some constant $c>0$.

Applying Bayes' rule as before, we obtain, for some other $c>0$,

$$
\begin{aligned}
& p(\mathbf{x} \mid \text { OBSERV }) \\
& =c \prod_{(R, L, t)} f(D(t)=\operatorname{SignaL}(t) \mid \mu, \sigma) p(R, L) p(\mathbf{x})
\end{aligned}
$$

Thus we can estimate the likelihood of the tag position from the observations as before.

\section{COORDINATION AND ACTIVE TRACKING}

Using the methods in Section IV and Section V, one may inferthe most likely spatial position of any given tag. However, the receivers work in an uncoordinated fashion by randomly settings their respective power levels. While, the system is stable in that it will always converge to the true position of the tag, the time response may be very slow, i.e. many observations are needed for the estimate $\mathrm{x}^{*}$ to converge. In this section, we show that one can dramatically improve the time response of the spatial inference algorithm by means intelligent tracking, i.e., control the power levels of individual receivers in a cooperative fashion.

The tracking strategy is characterized by $p(R, L)$, i.e. the amount of scans allocated to receiver and power level $L$. Since each receiver gathers samples independently, the samples allocated to each receiver is the same. Thus, we can write

$$
p(R, L)=p(L \mid R) \cdot p(R)=c \cdot p(L \mid R)
$$

One may think of $p(L \mid R)$ as the percentage of observations to be collections at power level $L$ at receiver $R$.

Recall from information theory, the amount of information obtained by some action is measured as the decrease of entropy of the randomness before and after the action. In our scenario, the action is the collection of $N$ observations. We wish to actively set the power levels using $p(L \mid R)$ so that the resulting entropy is minimized, thus maximizing the information gain. We assume in each epoch of observation, we always collection $N$ observations, and then compute $p(\mathbf{x} \mid$ OBSERV $)$. The active tracking algorithm is shown in Algorithm 3.

\section{Designing optimal scanning strategy}

Given $p(\mathbf{x} \mid$ OBSERV $)$, we can compute the entropy

$$
H=-\sum_{\mathbf{x}} p(\mathbf{x} \mid \text { OBSERV }) \log p(\mathbf{x} \mid \text { OBSERV })
$$

In order to design the scanning strategy $p(L \mid R)$, we need to estimate the spatial probability distribution for the next epoch. Let $p(\mathbf{x} \mid$ OBSERV $)$ be the distribution after the previous epoch, and $p^{\prime}(L \mid R)$ be the scanning strategy to be used in the next epoch. Again OBSERV is all the observations collected since the beginning. Denote $p^{\prime}\left(\mathbf{x} \mid \mathrm{OBSERV}^{\prime}\right)$ as the distribution after the next epoch. Then, $p^{\prime}\left(\mathbf{x} \mid \mathrm{OBSERV}^{\prime}\right)$ can be

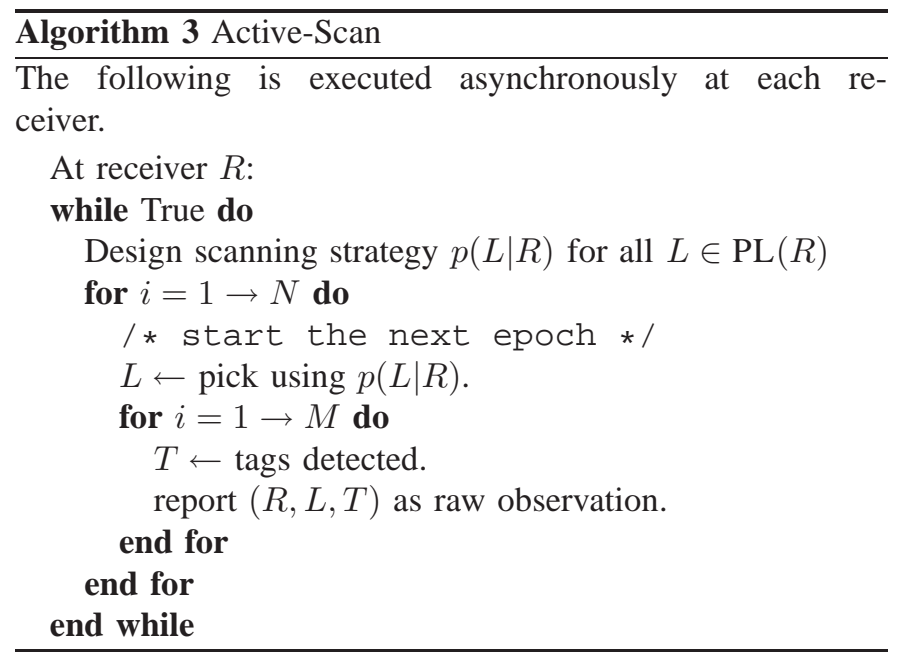

estimated by Equation 1 or Equation 3 by substituting $p(\mathbf{x})$ with $p(\mathbf{x} \mid$ OBSERV $)$. In both cases, $p^{\prime}\left(\mathbf{x} \mid \mathrm{OBSERV}^{\prime}\right)$ is of the form:

$$
p^{\prime}\left(\mathbf{x} \mid \text { OBSERV }^{\prime}\right)=c \prod_{(R, L)} a_{\mathbf{x}, R, L} p(L \mid R)^{k_{R, L}}
$$

where $a_{R, L}$ are constants, and $k_{R, L}$ are the number of distinct tags deleted by receiver $R$ at power level $L$. The estimated entropy of the next epoch is given by:

$$
\begin{aligned}
& H^{\prime}= c_{1} \sum_{\mathbf{x}} p^{\prime}\left(\mathbf{x} \mid \text { OBSERV }^{\prime}\right) \log p\left(\mathbf{x} \mid \text { OBSERV }^{\prime}\right) \\
&= c_{2} \sum_{\mathbf{x}}\left[\left(\prod_{(R, L)} a_{\mathbf{x}, R, L} p(L \mid R)^{k_{R, L}}\right)\right. \\
&\left.\sum_{(R, L)}\left(\log \left(a_{\mathbf{x}, R, L}\right)+k_{R, L} \log p(L \mid R)\right)\right]
\end{aligned}
$$

This allows us to obtain the optimal scanning strategy by solving the systems of equations:

For all receivers $R$ and their power levels $\operatorname{PL}(R)$ :

$$
\begin{aligned}
\frac{\partial H^{\prime}}{\partial p(L \mid R)} & =0 \\
\text { subject to: } \sum_{L \in \operatorname{PL}(R)} p(L \mid R) & =1
\end{aligned}
$$

One can verify that Equation 5 can be solved analytically with closed form solutions. We omit the details due to space limitation, and will defer the detailed derivations to the extended version of this article.

\section{IMPLEMENTATION AND EXPERIMENTS}

To verify the performance of the spatial inference framework presented Section IV-VI, we have setup four RFID receivers in our lab. The four receivers are wall mounted at the four corners of the room as shown in Figure 3. They are also connected to the central inference server via Ethernet LAN connection. The receivers report only deleted tags but not 


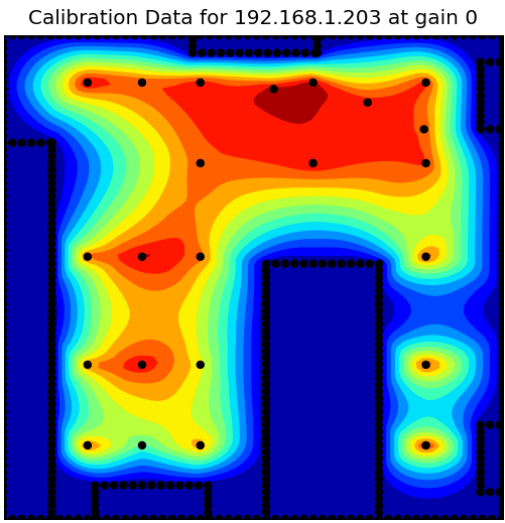

(a) Probability over space at highest power (b) level
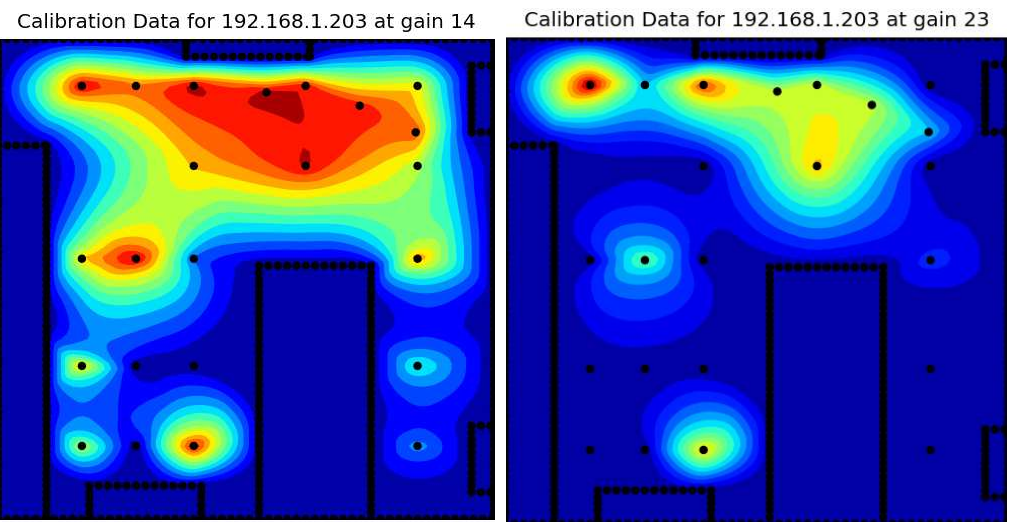

b) Probability over space at mid-power (c) Probability over space at low power level level

Fig. 2. The probability of detection across space of South-East (SE) receiver at different power levels.

their detected signal strength, thus we utilize the statistical model described in Section IV which do not require signal strength data. The individial power levels of each receiver can be remotely controlled by the inference server. Power level 0 is the strongest, while power level 31 is the weakest.

\section{A. Calibration}

In order to obtain the model parameters $\{\alpha(\mathbf{x}, R, L)\}$ in Equation 1, we perform calibration over a grid of spatial positions that are irregularlly spaced apart shown in Figure 3. A RFID tag is placed at each position, and the calibration algorithm in Algorithm 1. For efficiency, we execute the main loop in parallel.

Figure 4 show the change of detection rate of two different tag by the same receiver at its different power levels. As we can see that the detection rate drops as the power level decreases. Note, however, due to interference and signal echos, one may occasionally see increase of detection rate with decreasing power level. The inference algorithm is insensitive to these irregularities.

Figure 2 shows the detection rate over the lab room for a particular receiver at three different power levels. We see that, at high power level (Left in Figure 2), there is a good coverage of the room, with certain dead spots of poor detection. At low power level (Right in Figure 2), the coverage shrinks only to where the receiver is physically located. Again, due to the irregularity of the room, we see interesting patterns due to reflection of radio signals. Our inference algorithm is robust to these types of irregularities.

\section{B. Inference}

We compared the two inference algorithms: uniform scan and active scan. Both scanning strategies always correctly converge to the true position of the tag of interest. Figure 5 shows the result of the inference algorithm after 200 observations using uniform scan. The algorithm correctly converges to the true location of the tag. Each observation takes $\approx 0.25$ seconds, so it took 50 seconds for uniform scan to locate the tag.

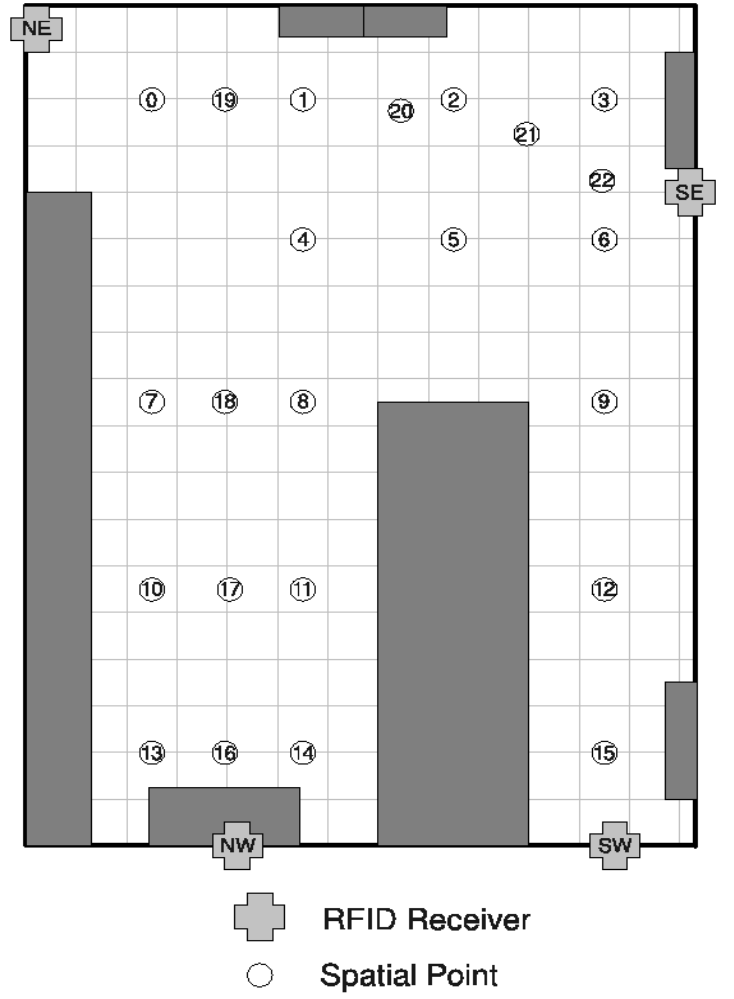

Fig. 3. Room layout

Active scan, in comparison, is must faster in its convergence rate. In order to compare the converage rates numerically, we calculate the entropy $H$ of the inferred distribution $p(\mathbf{x} \mid$ OBSERV $)$ each new observation received. As the inference algorithm converges to the true tag location, the entropy $H$ approaches 0 .

Figure 6 shows the average entropy curves for active scan and uniform scan over multiple inference runs. For each inference run, both algorithms converge to the true tag location. Note that the active scan converges using only half the number of readings. Thus, with only 100 readings $(\approx 25$ seconds), 


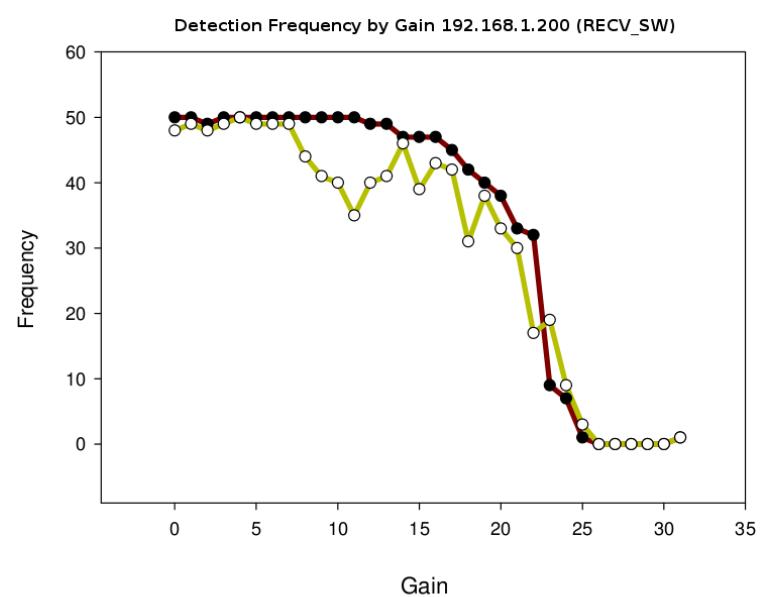

Fig. 4. Probability of detection over different power levels for two different receivers $R_{1}$ and $R_{2}$ with respect to the same tag located at fixed position. Note Gain 0 corresponds the strongest power level while gain 31 corresponds the weakest power level.

using active scan, one is able to infer the location of the tag of interest.

\section{CONCLUSION AND FUTURE WORK}

We have presented a statistical spatial inference framework to locate RFID tags using observations from a network of distributed RFID receivers. By means of model parameter estimation, our spatial inference method is highly robust to irregularities due to radio frequency interferences and environmental obstructions. Furthermore, by actively controlling the power levels of the RFID receivers, we are able to formulate an optimal detection strategy which we refer to as the active scan. Active Scan is offers the maximal information gain with the minimal number of observations, thus allows one to infer the true location of the tags of interest with minimal number of observations.

We have conducted experiences using four RFID receivers, and have demontrated the robustness and responsiveness of our approach.

Currently, we are in the process of acquiring additional RFID receivers that have other interesting physical characteristics - tunable anntenas, available signal strength, variable sampling rates. As future work, we plan to enrich our statistical models to take into account of these new controllable features of better RFID receivers. It is also interesting to investigate the inference problem when we have a network of heterogeneous RFID receivers.

\section{REFERENCES}

[1] Qiuxia Chen, Dik-Lun Lee, and Wang-Chien Lee. Rule-based wifi localization methods. In EUC '08: Proceedings of the 2008 IEEE/IFIP International Conference on Embedded and Ubiquitous Computing, pages 252-258, Washington, DC, USA, 2008. IEEE Computer Society.

[2] John Curtin, Robert J. Kauffman, and Frederick J. Riggins. Making the 'most' out of rfid technology: a research agenda for the study of the adoption, usage and impact of rfid. Inf. Technol. and Management, 8(2):87-110, 2007.

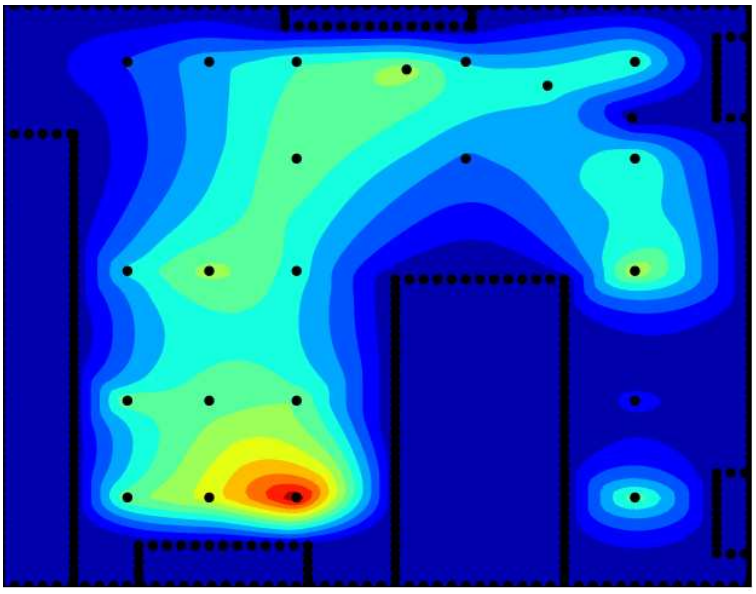

Fig. 5. Inference of a tag position after 200 observations.

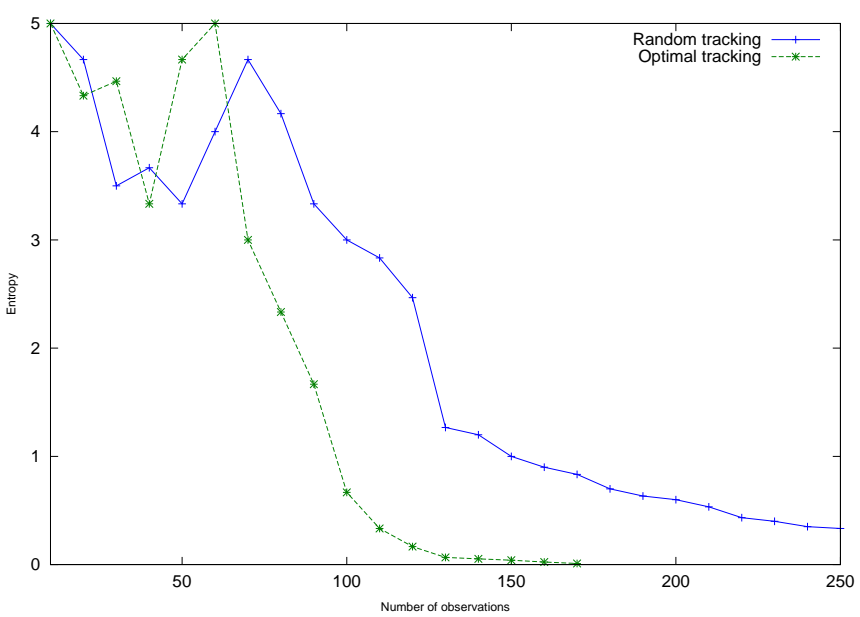

Fig. 6. Performance of random tracking versus optimal tracking

[3] Peter D. DeVries. The state of rfid for effective baggage tracking in the airline industry. Int. J. Mob. Commun., 6(2):151-164, 2008.

[4] InformationWeek. Gillette razors get new edge: Rfid tags. InformationWeek, January 13, 2003 Issue.

[5] Vlad Krotov and Iris Junglas. Rfid as a disruptive innovation. J. Theor. Appl. Electron. Commer. Res., 3(2):44-59, 2008.

[6] Dik Lun Lee and Qiuxia Chen. A model-based wifi localization method. In InfoScale '07: Proceedings of the 2nd international conference on Scalable information systems, pages 1-7, ICST, Brussels, Belgium, Belgium, 2007. ICST (Institute for Computer Sciences, Social-Informatics and Telecommunications Engineering).

[7] Adam Melski, Lars Thoroe, and Matthias Schumann. Managing rfid data in supply chains. Int. J. Internet Protoc. Technol., 2(3/4):176-189, 2007.

[8] Lionel M. Ni, Yunhao Liu, Yiu Cho Lau, and Abhishek P. Patil. LANDMARC: Indoor location sensing using active rfid. Wireless Networks, 10(6):701-710, 2005.

[9] George Roussos and Vassilis Kostakos. rfid in pervasive computing: State-of-the-art and outlook. Pervasive Mob. Comput., 5(1):110-131, 2009.

[10] Lasse Thiem, Björn Riemer, Marcus Witzke, and Thomas Luckenbach. Rfid-based localization in heterogeneous mesh networks. In SenSys '08: Proceedings of the 6th ACM conference on Embedded network sensor systems, pages 415-416, New York, NY, USA, 2008. ACM.

[11] Yu-Ju Tu and Selwyn Piramuthu. Reducing false reads in rfid-embedded supply chains. J. Theor. Appl. Electron. Commer. Res., 3(2):60-70, 2008. 